\title{
A Voltage Stability Preventive Control Approach based on Generator Reactive Power Reserve Sensitivities
}

\author{
J. Zhao, Y. Rao \\ College of Energy and Electrical Engineering \\ Hohai University \\ Nanjing, China
}

\begin{abstract}
The generator reactive power reserve is closely related to the power system voltage stability. The voltage stability margin can be effectively improved by improving the critical generator reactive power reserves. A static stability preventive control approach based on reactive power reserve sensitivities is presented. The proposed approach can be divided into a sensitivity computation sub-problem and a sensitivity-based quadratic programming optimization sub-problem. In the previous sub-problem, critical generators are identified by calculating the load margin and sensitivities of generator reactive power reserve are calculated. In the latter sub-problem, the most effective control actions are selected to improve the computation efficiency of quadratic optimization problem. Simulation results on IEEE 30-bus power system indicate that the proposed method is effective.
\end{abstract}

Keywords-generator reactive power reserve; preventive control; generator exciting current limit; reactive power reserve sensitivity; quadratic programming

\section{INTRODUCTION}

With the expansion of the scale of power grid and the increase of load, the operating point of power system is much closer to the boundary of the stability operation and voltage stability problem becomes severer. The main tasks of power system stability analysis include the voltage stability margin calculation, contingency selection and ranking, voltage stability optimization control.

The solutions of the preventive control problem can be classified as the integration method and the decompositioncoordination method[1-2]. In the former method, the optimal power flow under several contingencies simultaneously is solved by using the nonlinear programming interior point method or the Newton method. However, the Hessian matrix needs to be formed, which is a computational demanding work. In the latter method, the preventive control problem can be divided into the voltage stability margin computation subproblem and the corresponding sensitivities and optimization control sub-problem. Although the computation speed is slow and many intermediate results appeared, the algorithms of the subtasks are mature which guarantees its robustness. Each task can be assembled by various models, which can be extended easily and the multiple-contingency coordinated control can be considered. A sensitivity calculation method of the voltage collapse point based on continuation power flow is proposed in [3-4], which has been known as a fast method of recognizing effective control. A linear optimization model of preventive and corrective control is built by using the margin sensitivity [5-6]. In [7], a sensitivity calculation method of the virtual voltage collapse point based on the contingency parameterization continuation power flow is proposed. The sensitivities of contingency instability margin with respect to controls are obtained. In [8], a liner programming model is established by using the proposed sensitivity. The candidate control group strategy and the controllable region limitation strategy are used to improve the computation efficiency of liner programming problem. In [9], an on-line preventive control algorithm for static voltage stability is presented, in which the generation power distribution factor for coming future is introduced as control parameters for static voltage stability margin. The feature of this algorithm is that serious contingencies and unstable contingencies can be solved in the same mathematical mode. In above methods, the precondition of load margin sensitivity calculation is that the Jacobian matrix of the power flow equation at the voltage collapse point is singular or leads to conversion to limited induced bifurcation. If the step size is too large, the critical point calculated by the continuation power flow is far away from the true bifurcation point, which causes inaccuracy of sensitivity calculation.

The amount of generator reactive power reserves(GRPR) is a measure of the degree of voltage stability [10]. It is critical to keep GRPR to maintain voltage stability [11]. In [12], statistical multi-linear regression models are utilized to transform the variations of system's reactive power reserves into direct information about voltage stability margin.

Voltage stability margin(VSM) can be enhanced by improving generator reactive power reserves. In [13], a reactive reserve-based contingency constrained optimal power flow method for the enhancement of voltage stability margins is proposed. In [14], an optimized reactive reserve management scheme based on the optimal power flow is proposed. In [15], a method based on the relationship between generator reactive power reserves and voltage stability margin is presented. The voltage stability margin is improved by using generator reactive power reserve sensitivities. The calculation of GRPR sensitivities is simple and intuitive. However, the unstable contingencies are not been considered. Using the sensitivity calculation in reference [15], a voltage stability 
preventive control approach based on generator reactive power reserve sensitivities is proposed. The system's voltage stability margin of post-contingency is improved indirectly by improving generator reactive power reserve.

\section{FORMULATION OF PREVENTIVE CONTROL PROBLEM}

In engineering, the GRPR is an important index to judge system's voltage stability, critical generator reactive power reserves have an approximate liner relationship with system's voltage stability margin ${ }^{[10]}$ :

$$
\lambda \cong K \sum_{i=1}^{n_{g}} Q_{r i}+b=K Q_{r}^{\text {Total }}+b
$$

where $n_{g}$ is the amount of critical generators, $Q_{r}^{\text {Total }}$ is the sum of critical generator reactive power reserves, $K$ is the ratio of change in system's voltage stability margin and change in critical generator reactive power reserves, $b$ is a constant.

Therefore, the formulation of preventive control problem using the system's reactive power reserves as voltage stability indicator can be written as:

$$
\begin{array}{lll}
\min & C(u) & \\
\text { s.t. } & f\left(x, u, \lambda_{0}\right)=0 & \\
& f_{i}\left(x_{i}, u, \lambda_{0}\right)=0 & \\
& Q_{r}(x, u) \geq Q_{r}^{\min } & \\
& Q_{r, i}\left(x_{i}, u\right) \geq Q_{r, i}^{\min } & \\
& h\left(x_{i}, u, \lambda_{0}\right) \leq 0 & \\
& h_{i}\left(x_{i}, u, \lambda_{0}\right) \leq 0 & \\
&
\end{array}
$$

where $f$ denote the load flow equations of base case, $f_{i}$ denote the load flow equations of $i$ th post-contingency system, $Q_{r}$ denotes GRPR of base case, $Q_{r, i}$ denotes GRPR of $i$ th post-contingency system. $Q_{r}^{\min }$ is the required minimum GRPR of base case, $Q_{r, i}^{\min }$ is the required minimum GRPR of $i$ th post-contingency system. $h$ denote the operational constraints of base case such as the voltage limits, $h_{i}$ denote the operational constraints of $i$ th post-contingency system.

\section{GENERATOR REACTIVE POWER RESERVES AND SENSITIVITY}

\section{A. The definition of Generator Reactive Power Reserve}

The definition of generator reactive power reserve is:

$$
Q_{r i}=Q_{g \max _{i}}\left(P_{g i}\right)-Q_{g i}
$$

where $Q_{r i}$ is the amount of reactive power reserve in generator $i, P_{g i}$ is the

current active power produced by generator $i, Q_{g \max _{i}}\left(P_{g i}\right)$ is the maximum reactive power limit given by the capability curve, $Q_{g i}$ is the current reactive power produced by generator $i$.

For non-salient pole generator, the maximum reactive power output

considering the maximum excitation current is:

$$
Q_{g \text { max }_{i}}\left(P_{g i}\right)=\frac{1}{X_{d i}}\left(\sqrt{\left(I_{f d i}^{\lim }\right)^{2} V_{g i}^{2}-P_{g i}^{2} X_{d i}^{2}}-V_{g i}^{2}\right)
$$

where $X_{d i}$ is generator d-axis reactance, $V_{g i}$ is generator's terminal voltage, $I_{f d i}^{\lim }$ is the maximum excitation current limit.

When low amounts of generator reactive power reserve and voltage stability margin are observed, a set of control $\Delta u$ need to be obtained to make generator reactive power reserve larger than the required value. The system's voltage stability margin is also improved as the increase of generator reactive power reserve.

\section{B. Generator Reactive Power Reserve Sensitivities Calculation}

Generator reactive power reserve sensitivities with respect to various controls are calculated as follows:

$$
\begin{aligned}
S_{i k}^{t} & =\frac{\partial Q_{r i}}{\partial u_{k}}=\frac{\partial Q_{\max _{i}}\left(P_{g i}\right)}{\partial u_{k}}-\frac{\partial Q_{g i}}{\partial u_{k}} \\
& =\frac{\partial Q_{\max _{i}}\left(P_{g i}\right)}{\partial u_{k}}-\sum_{j=1}^{n}\left(\frac{\partial Q_{t i}}{\partial \theta_{j}} \frac{\partial \theta_{j}}{\partial u_{k}}+\frac{\partial Q_{t i}}{\partial V_{j}} \frac{\partial V_{j}}{\partial u_{k}}\right)
\end{aligned}
$$

where $S_{i k}^{t}$ represents the sensitivity of the reactive power reserve of generator $i$

with respect to $k$ th control variable of $t$ th contingency, $u_{k}$ is the $k$ th control

variable, $Q_{t i}$ is the injected reactive power at node $i$, bus voltage angles and magnitudes of $t$ th contingency are given by $\theta_{j}$ and $V_{j}$.

According to equation (4), if the control type is active power generation,

$$
\frac{\partial Q_{\max _{i}}\left(P_{g i}\right)}{\partial u_{k}}=-\frac{P_{g i}}{\sqrt{\left(I_{f d i}^{\lim }\right)^{2} V_{g i}^{2} / X_{d i}^{2}-P_{g i}^{2}}}
$$

If the control type is shunt capacitors switch or load shedding,

$$
\frac{\partial Q_{\max _{i}}\left(P_{g i}\right)}{\partial u_{k}}=0
$$

The reactive power injection equation at node $i$ is: 


$$
Q_{t i}=V_{i} \sum_{j=1}^{n} V_{j}\left(G_{i j} \sin \theta_{i j}-B_{i j} \cos \theta_{i j}\right)
$$

$\frac{\partial Q_{t i}}{\partial \theta_{j}}$ and $\frac{\partial Q_{t i}}{\partial V_{j}}$ can be obtained from the differentiation of the reactive power injection equation at node $i$.

The power flow equation can be described as $f(x, u)=0$, then:

$$
S_{x u}=-\left[\frac{\partial f}{\partial x}\right]^{-1}\left[\frac{\partial f}{\partial u}\right]=-J^{-1}\left[\frac{\partial f}{\partial u}\right]
$$

where $S_{x u}$ represents the sensitivity of the state variables with respect to various control variables, $J$ is the Jacobian matrix of power flow.

\section{Recognition of Critical Generators}

The severe deficiency of some critical generators will lead to voltage collapse. If the load is increased to the collapse point, the generator's reactive power reserve is exhausted. Then the generator is recognized as critical generator.

\section{QUADRATIC PROGRAMMING OPTIMUM CONTROL PROBLEM}

In this paper, a quadratic programming optimum control problem is formed based on the generator reactive power reserve sensitivities to obtain the optimum control solution.

$$
\begin{gathered}
\min \sum_{i=1}^{n_{t}} w_{i} \sum_{k=1}^{n_{i}}\left(c_{k} \Delta u_{k}\right)^{2} \\
\text { s.t. } \sum_{t=1}^{n_{c t}} \sum_{k=1}^{n_{c}} S_{i k}^{t} \Delta u_{k} \geq \alpha Q_{r, i}^{\min }-Q_{r, i} \\
\underline{V}_{m} \leq \sum_{k=1}^{n_{c}} S_{V, m k} \Delta u_{k}+V_{m} \leq \bar{V}_{m} \\
u_{k}^{\min }-u_{k}^{0} \leq \Delta u_{k} \leq u_{k}^{\max }-u_{k}^{0}
\end{gathered}
$$

where $n_{t}$ is the number of control type, $w_{i}$ is the weighting factor corresponding to control type, $n_{i}$ is the control number of control type $i, c_{k}$ is the cost factor of $k$ th control variable. The determination of the weighting factor should reflect the priority of different control types. The lower the control priority level is, the bigger the weighting factor is. The first level is shunt capacitor switch, the second level is the readjustment of generator real power, the third level of control is load shedding. $n_{c}$ is the number of control variable, $\alpha$ is the compensation factor which is used for improve the effectiveness of liner sensitivities(say 1.03-1.05). In equation (12), $S_{V, m k}$ represents the sensitivity of voltage with respect to various control variables, $\underline{V}_{m}$ and $\bar{V}_{m}$ are upper and lower limits of variable $V_{m}$ respectively, $u_{j}^{\max }$ and $u_{j}^{\min }$ are upper and lower limits of variable $u_{j} \cdot u_{k}^{0}$ is the initial amount of control variable $u_{k}$. The first inequality constraints represents the critical generator reactive power reserves will be brought back to their minimum reserve limits. The second inequality represents voltage limits. The third inequality constraints enforce limits on the control variables in order to make sure that the amount of control is within physical and operational limits.

First, the severe contingencies should be found. If the VSM is below the required value, critical GRPR sensitivities should be calculated. Then, select the most sensitive controls into the candidate control set and solve the quadratic programming optimum control problem. At last, enforce the obtained control actions and update. At the first iteration, the ratio of change in system's voltage stability margin and change in critical generator reactive power reserves is set to be a large value $(K=10000)$. After the first round of control, it can be obtained by dividing the change in voltage stability margin by the sum of the changes in all critical generator reactive power reserves.

\section{SiMULATION RESULTS}

The proposed preventive control method is used in IEEE 30-bus system. The studied scenario is a high load case.

Reactive power reserves of generator 8, 11, 13 are lower than the required values, which need to be improved. Under contingency 1 , after stressing the system to the collapse point, $Q_{r 13}$ has its limits reached and loses voltage control capability, thus generator 13 is recognized as critical generator.

Table 1 shows severe contingencies and preventive control process. The minimum voltage stability margin is 0.46 . For a severe contingency, six shunt capacitors, one generator active power adjustment and four load shedding controls with top sensitivities are selected into the candidate controls. The weighting factors of 3 levels are set to 1,50 and 50 respectively. The compensation factor is set to 1.03 .

TABLE I. LOAD LEVEL OF SEVERE CONTINGENCIES IN THE PROCESS OF PREVENTION CONTROL.

\begin{tabular}{llll}
\hline & Initial & Iteration 1 & Iteration 2 \\
\hline Contingency1 & 0.2827 & 0.3383 & 0.4829 \\
\hline Contingency2 & 0.4694 & 0.4961 & 0.5837 \\
\hline Base case & 1.2287 & 1.2903 & 1.4616 \\
\hline
\end{tabular}

The amount of shunt capacitor is far larger than load shedding and generator active power due to the weighting factors. From table 1, the voltage stability margin of base case and contingencies is increasing as the process of control. Generator reactive power reserves are apparently improved. The base case voltage magnitudes in the process of control are in 0.9-1.1p.u.

\section{CONCLUSION}

In the paper, a voltage stability preventive control approach based on GRPR sensitivities is proposed. By using the quadratic programming technique, the preventive control approach can be divided into a sensitivity computation sub- 
problem and a sensitivity-based optimization sub-problem. In the previous sub-problem, critical generators are identified by calculating load margin and sensitivities of GRPR are calculated. In the latter sub-problem, the most effective control actions are selected to improve the computation efficiency of quadratic optimization problem. Simulation results on IEEE 30-bus power system indicate that the proposed method is effective.

\section{ACKNOWLEDGEMENTS}

This work is supported by National Natural Science Foundation of China (51077042);

The National High Technology Research and Development Program of China(863 Program) (2012AA050207); Special Foundation of The doctoral program of Higher

Education (20120094110008).

\section{REFERENCE}

[1] Wang, X., Ejebe, G.C. \& Tong, J., et al. Preventive/corrective control for voltage stability using direct interior point method. IEEE Trans on Power System, 13(3), pp. 878-883, 1998.

[2] Canizares, C.A., Rosehart, W. \& Berizzi, A., et al. Comparison of voltage security constrained optimal power flow techniques. IEEE/PES Summer Meeting, Vancouver, BC, Canada, pp. 1680-1685, 2001.

[3] Greene, S., Dobson, I. \& Alvarado, F.L. Sensitivity of the loading margin to voltage collapse with respect to arbitrary parameters. IEEE Trans on Power Systems, 12(1), pp. 262-272, 1997.

[4] Greene, S. Dobson, I. \& Alvarado, F.L. Sensitivity of transfer capability margins with a fast formula. IEEE Trans on Power Systems, 17(1), pp. 34-40, 2002.

[5] Feng, Z., Ajjarapu, V. \& Maratukulam, D.J., A comprehensive approach for preventive and corrective control to mitigate voltage collapse. IEEE Trans on Power Systems, 15(2), pp. 791-797, 2000.

[6] Capitanescu, F. \& Custem, T.V., Preventive control of voltage security margins: a multicontingency sensitivity-based approach. IEEE Trans on Power Systems, 17(2), pp. 358-364, 2002.

[7] Zhao, J., Chiang, H. D. \& Li, H., et al. A new contingency parameterization CPF model and sensitivity method for voltage stability control, IEEE PES General meeting, July, San Francisco, USA, pp. 376382, 2005.

[8] Zhao, J., Chiang, H. D. \& Li, H., et al. A Novel Preventive Control Approach for Mitigating Voltage Collapse, IEEE PES General meeting, July, Montréal, Canada. pp.1-6, 2006.

[9] Zhong, H., Wu, Z., \& Zhang, X., et al. Preventive control for static voltage stability based on generation power distribution factor. Proceedings of the CSEE, 31(34), pp. 150-155, 2011. (in Chinese)

[10] Bao, L., Huang, Z. \& Xu, W., Online voltage stability monitoring using VAR reserves. IEEE Trans on Power Systems, 18(4), pp. 1461-1469, 2003.

[11] Wu, Hao., Guo, R. \& Gan, D., et al. Analysis and computation of effective reactive power reserve of generators. Automation of Electric Power Systems, 35(15), pp. 13-17, 2011. (in Chinese)

[12] Leonardi, B. \& Ajjarapu, V., Development of multiliner regression models for online voltage stability margin estimation. IEEE Trans on Power Systems, 26(1), pp. 374-383, 2011.

[13] Song, H., Lee, B., Kwon, S.H. \& Ajjarapu, V., Reactive reserve-based contingency constrained optimal power flow (RCCOPF) for enhancement of voltage stability margins. IEEE Trans on Power Systems, 18(4), pp. 1538-1546, 2003.

[14] Dong, F., Chowdhury, B.H. \& Crow, M.L., et al. Improing voltage stability by reactive power reserve management. IEEE Trans on Power Systems, 20(1), pp. 338-345, 2005.

[15] Leonardi, B. \& Ajjarapu, V., An approach for real time voltage stability margin control via reactive power reserve sensitivities. IEEE Trans on 